\title{
Characterization of dielectric spheres by spiral imaging
}

\author{
Dmitri Petrov, ${ }^{1,2, *}$ Nicolas Rahuel, ${ }^{1,3}$ Gabriel Molina-Terriza, ${ }^{4}$ and Lluis Torner ${ }^{1}$ \\ ${ }^{1}$ ICFO-Institut de Ciencies Fotoniques and Universitat Politecnica de Catalunya, 08860 Castelldefels (Barcelona), Spain \\ ${ }^{2}$ ICREA-Institució Catalana de Recerca i Estudis Avancats, 08010 Barcelona, Spain \\ ${ }^{3}$ Ecole Nationale Superieure de Physique de Strasbourg, F-67412 Illkirch-Cedex, France \\ ${ }^{4}$ QsciTech and Department of Physics \& Astronomy, Macquarie University, 2109 New South Wales, Australia \\ *Corresponding author: Dmitri.Petrov@icfo.es
}

Received September 29, 2011; revised December 21, 2011; accepted January 17, 2012; posted January 18, 2012 (Doc. ID 155529); published February 24, 2012

\begin{abstract}
We study the spiral spectra scattered off transparent dielectric spheres when probed by different Laguerre-Gaussian light beams, carrying nested topological wavefront dislocations. We show that such scattering data may be employed to determine geometrical properties of the spheres, such as their position. The technique is a generalization of standard Mie scattering, and it can be extended to study and to characterize nanospheres. @ 2012 Optical Society of America
\end{abstract}

OCIS codes: $\quad 080.4865,290.4020$.

Light beams carry angular momentum, which can be divided in two components, namely, orbital angular momentum (OAM) and spin angular momentum (SAM). In the case of paraxial fields, the two contributions can be separated and thus, independently prepared and measured [1]. The SAM, i.e., the polarization of light, is routinely used to characterize materials in ellipsometry and in several other applications in metrology. It is not always properly appreciated that the OAM may also be used to probe geometrical properties of objects $[2,3]$ using the unique properties of the topological wavefront dislocations, or optical vortices, associated to light beams carrying OAM. The technique is based on the elucidation and interpretation of the relevant parts of the so-called spiral spectra of the light beams scattered off objects when illuminated by different probing beams. In this Letter we study several spiral spectral modes scattered by paraxial transparent dielectric spheres when probed by LaguerreGaussian (LG) modes. By and large, the spiral spectrum may be used to elucidate different geometrical properties of spheroids, such us their size, position, and ellipticity, as well as the refractive index contrast between the particle and the surrounding medium. Here we focus on how the spiral spectra vary with the position and size of spherical polystyrene beads.

Our setup may be viewed as a generalization of standard Mie scattering [ [4] , including its application to complex geometries [5]. Mie scattering of incident beams carrying OAM was studied in [6,7], where the spatial distribution of the scattered intensity was used to monitor properties of microparticles. Other previous studies have focused on the effect of the light field on the illuminated particles to analyze the SAM-to-OAM conversion in focused beams [8]. In our approach we extract the information encoded in the OAM eigenmodes of the paraxial wave to be detected, $F(\mathrm{Q}, \phi, z)[2,3]$ and use it to infer geometrical properties of the spheres. For simplicity we consider a single linear polarization of the paraxial electrical field, but our technique may be readily extended to multiple polarizations.

Using the setup sketched in Fig. 1, we probed several spheres with light generated by a He-Ne laser $(633 \mathrm{~nm})$. A beam coupled to a monomode fiber, in order to obtain a mode with azimuthal index $m_{i}=0$, was transformed onto different LG modes with azimuthal indices $m_{i}=$ +1 , i.e., $\mathrm{LG}_{0,1}$, and with index $m_{i}=-1$, i.e., $\mathrm{LG}_{0,-1}$, by using computer-generated blazed holograms $\left(\mathrm{CGH}_{1}\right.$, $\mathrm{CGH}_{2}$ ).

The resulting LG beams, with $m_{i}=-1,0,1$, were focused by an oil-immersion microscope lens $\mathrm{O}_{1}$ with $\mathrm{NA}=1.25$ onto a sample, which consisted of two $80 \mu \mathrm{m}$ coverslips separated by a $100 \mu \mathrm{m}$ gap filled by water with a mixture of polystyrene spheres of different diameters. The spheres that stuck on the coverslip surfaces were the ones used in the experiments. In order to choose a single sphere as a scatterer, we used a white light source and a $\mathrm{CCD}$ camera $\left(\mathrm{CCD}_{1}\right)$ to image the chosen target. The position of the selected sphere was controlled by a submicrometer precision piezostage. The incident Gaussian beam had an FWHM at the target position of around $0.4 \mu \mathrm{m}$, while the size of the LG modes used was around $1.5 \mu \mathrm{m}$. The optical field scattered by the sphere together with the nonscattered part of the incoming field was collimated by a microscope objective $\mathrm{O}_{2}$ with $\mathrm{NA}=0.65$. With a flip mirror mount, FM, we were able to study the total output field distribution with a CCD camera $\left(\mathrm{CCD}_{2}\right)$ or to analyze its spiral spectrum. The components of the OAM modes of the output field

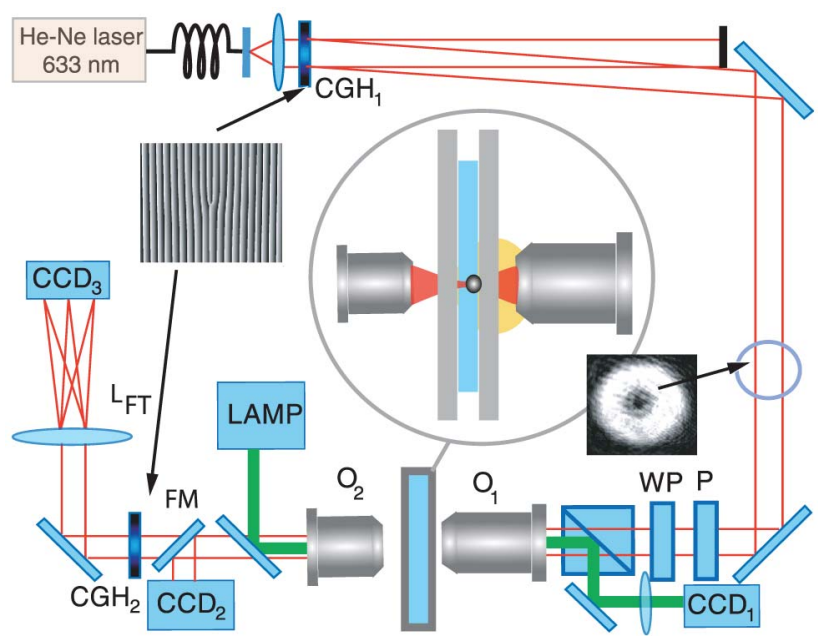

Fig. 1. (Color online) Experimental setup. See text for details. 
were analyzed with a combination of a CGH $\left(\mathrm{CGH}_{2}\right)$ followed by a lens $\mathrm{L}_{\mathrm{FT}}$ and a $\mathrm{CCD}$ camera $\left(\mathrm{CCD}_{3}\right)$.

Such a measuring system records the Fourier transformed diffraction orders coming out from the hologram. The central point of each of the diffraction orders provides key information about the OAM content of the scattered field [3]. In particular, the central point intensity of the Fourier transformed order $m$ can be expressed as $M_{m}\left(z_{0}\right)=2 \pi\left|\int_{0}^{\infty} \operatorname{d\varrho } \rho A_{m}\left(\mathrm{Q}, z_{0}\right)\right|^{2}, \quad$ where $\quad A_{m}(\mathrm{Q}, z)=$ $\int_{0}^{2 \pi} \mathrm{d} \phi F(\mathrm{Q}, \phi, z) \exp (-i m \phi) / \sqrt{2 \pi}$ [9]. Importantly, notice that this quantity is not equivalent to the power carried by the mode, i.e., $C_{m}=\int_{0}^{\infty} \mathrm{d} \varrho\left|A_{m}(\mathrm{Q}, z)\right|^{2}$, but because $M_{m} \leq C_{m}$, when properly normalized $M_{m}$ provides a lower bound to the energy of the modes. This method is simple and very robust and in many cases is an advantageous alternative to other techniques used elsewhere [10-14].

The measuring system was calibrated by displacing the sphere far from the incident beam so that the position of the center of the diffraction order could be readily obtained. Once the coordinates of the corresponding pixels were found, in a typical experiment, the sphere was moved through the incident beam continuously, grabbing at the same time a sequence of images of the diffraction orders. We then extracted the intensity of the central pixels. We analyzed several orders at the same time, thus extracting the spiral spectrum $M_{m}$.

We probed spheres with diameters ranging from 1 to $20 \mu \mathrm{m}$. The central features of the collected data are presented in Fig. 2. The rows of the plot depict the data for each analyzed mode, namely $m=-1,0,+1$. Thus, in (a)
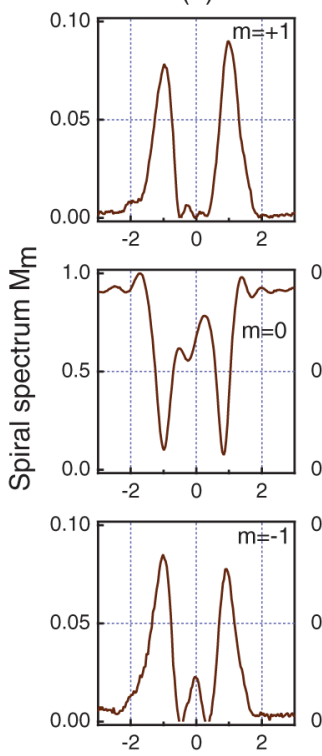

(b)
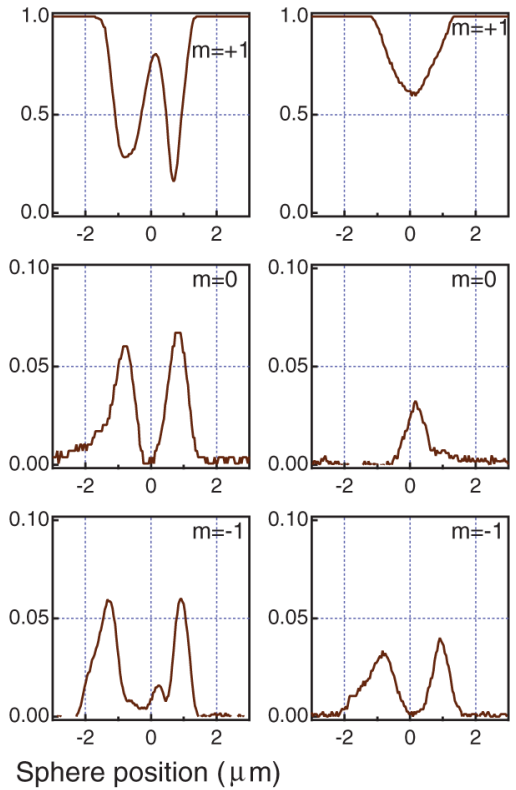

Fig. 2. (Color online) Dependence of the spiral spectra in the total scattering field versus the sphere position for three spiral modes $m=+1, m=0$, and $m=-1$. The sphere diameter is $2 \mu \mathrm{m}$ in columns (a) and (b) and $1 \mu \mathrm{m}$ in (c). Solid lines represent linearly polarized Gaussian (a) or $\mathrm{LG}_{0,1}$ (b), (c) input beams. In column (b), the results with linear, left- and rightpolarized input beams showed practically the same dependence. All modes normalized to the maximum value of the output mode $m=m_{i}$.
Fig. 2, column (a), we show the results obtained by probing a $2 \mu \mathrm{m}$ diameter sphere with a Gaussian beam $\left(m_{i}=0\right)$. The results show the transformation of the input beam from a pure mode to a superposition of modes. In particular, when the sphere is displaced by half its diameter, the contribution of the mode $m=0$, shown in the central panel, decreases significantly, while the contribution of the other modes increases. Note that the asymmetry in the mode distributions when displacing the sphere toward one or the other direction ought to be attributed to experimental inaccuracies of the positioning system and to small asymmetries of the focused beam due to lens aberrations. Transfer between different modes can also be observed when the incident beam is a pure mode with $m_{i}=1$. Such is the case is shown in Fig. 2, column (b). Similar results were obtained for input modes with $m_{i}=-1$, and with different polarizations.

The transfer of OAM modes changes drastically when decreasing the size of the sphere, as in Fig. 2, column (c), which corresponds to a sphere of $1 \mu \mathrm{m}$ diameter. Note that the sphere is smaller than the size of the input beam, which in this case is again an $\mathrm{LG}_{0,1}$ mode. The two lobes disappear, and in the analyzed mode $m=1$ we only observe a decrease of $M_{1}$ when the sphere is exactly on axis. The transfer of OAM goes primarily to the mode $m=0$, which shows a peak when the displacement is zero. There is also a contribution to the higher mode $m=-1$, which is maximum when the sphere is displaced by $1 \mu \mathrm{m}$. A similar result is observed for the $2 \mu \mathrm{m}$ sphere in Figs. 2, columns (a) and (b), but the effect is much smaller than the off-axis transfer. Note that, as mentioned above, the results presented give a lower bound to the energy flow carried by the mode. This means that when the sphere is on axis, there is a transfer of energy when $M_{m \neq m_{i}} \neq 0$. For spheres of larger diameters, we

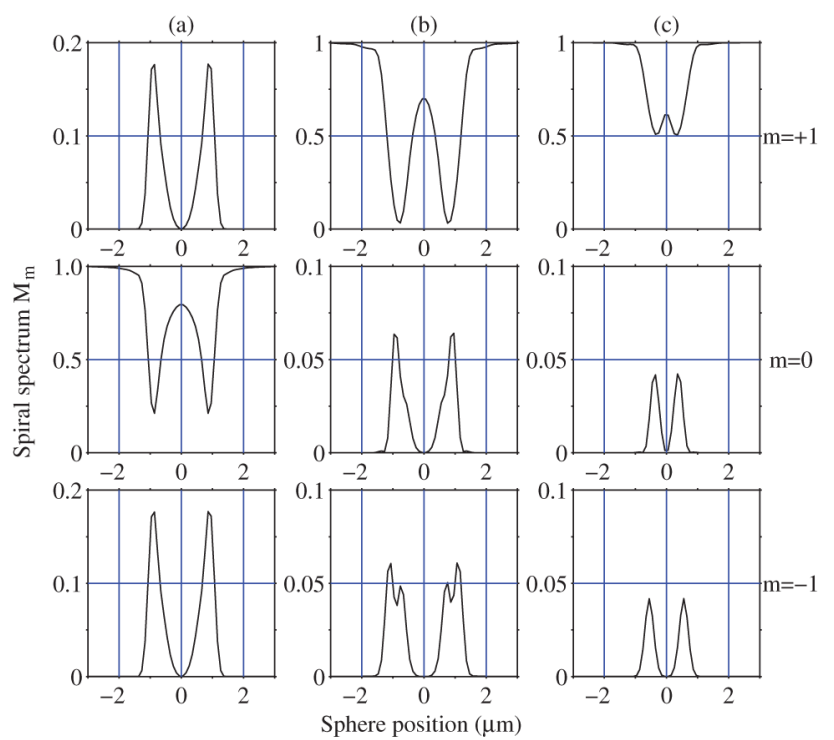

Fig. 3. (Color online) Results of numerical simulations of the mode transformation efficiency when the incident wave is the Gaussian beam [(a)] and the LG beam [(b), (c)]. The size of the sphere is $2 \mu \mathrm{m}$ [(a), (b)] and $1 \mu \mathrm{m}$ [(c)]. The spiral spectra of the modes transformed due to the Mie scattering are normalized on the maximal value of the spiral spectrum of the incident mode. 
observed a similar behavior similar than for 1 and $2 \mu \mathrm{m}$ spheres, except that when the incident beam passed far from the edges of the sphere, the spiral spectra amplitudes oscillate with the sphere shift with the period of oscillations depending on the spiral mode index. We attribute such oscillations to interference effects.

We analyzed the scattering data using standard scalar Fourier optics. In the calculations, the effects of the sphere is modeled as a change of phase of the input beam by $\Delta \phi=2 k\left(n_{s}-n_{a}\right) \sqrt{r_{s}^{2}-(x-d)^{2}-y^{2}}$, where $k$ is the wave number of the beam in vacuum, $n_{s}$ and $n_{a}$ are the refractive indices of the sphere and the medium, $r_{s}$ is the radius of the sphere, and $d$ is the displacement of the sphere with respect to the center of the beam in the $(x-y)$ plane. In Fig. 3 we present the calculations that correspond to the experimental results depicted in Fig. 2. The calculations display most of the features of the experiments for $2 \mu \mathrm{m}$ spheres, but they do not capture the transfer of modes in the on-axis case, which is dominant in the $1 \mu \mathrm{m}$ case. The discrepancy comes from the fact that the off-axis mode transfer is associated with a phase jump; thus, in order to capture the mode transfer in the on-axis case, one has to resort to a full vectorial analysis.

Notice the differences in the scattering data when the illuminating beam is a vortex-free Gaussian beam or a LG mode carrying nested topological vortices. Compare, for example, Figs. 2(a) $m=0$ and 2(b) $m=+1$. One readily observes that the central peak gets narrower when using an LG mode as the probe beam, a property that is linked to the scattering of the topological dislocations off the sphere and thus increases the sensitivity when measuring the position of the sphere close to the on-axis position. Similarly, measuring the spiral spectrum can give us more accurate information about the off-axis sphere position. These results are in agreement with results obtained addressing the inverse problem in generalized Mie theories $[15,16]$.

We focused on determination of the position of the spheres, but the technique provides information about different properties of the target objects. Of particular in- terest is the generalization of the technique to nanoscale objects at the nonparaxial regime based on the corresponding mode decomposition put forward in [17].

This work has been partially supported by the Ministry of Science and Innovation, Government of Spain, grants FIS2009-09928 and FIS2008-00114, the Australian Research Council project DP110103697, and by Fundació Cellex Barcelona.

\section{References}

1. L. Allen, M. W. Beijersbergen, R. J. C. Spreeuw, and J. P. Woerdman, Phys. Rev. A 45, 8185 (1992).

2. L. Torner, J. P. Torres, and S. Carrasco, Opt. Express 13, 873 (2005).

3. G. Molina-Terriza, L. Rebane, J. P. Torres, L. Torner, and S. Carrasco, J. Eur. Opt. Soc. 2, 07014 (2007).

4. C. F. Bohren and D. R. Huffman, Absorption and Scattering of Light by Small Particles (Wiley, 1983).

5. G. Gouesbet, J. Quant. Spectrosc. Radiat. Transfer 110, 1223 (2009).

6. A. S. van de Nes and P. Torok, Opt. Express 15, 13360 (2007).

7. V. Garbin, G. Volpe, E. Ferrari, M. Versluis, D. Cojoc, and D. Petrov, New J. Phys. 11, 013046 (2009).

8. Y. Zhao, J. S. Edgar, G. D. M. Jeffries, D. McGloin, and D. T. Chiu, Phys. Rev. Lett. 99, 073901 (2007).

9. G. Molina-Terriza, J. P. Torres, and L. Torner, Phys. Rev. Lett. 88, 013601 (2002).

10. J. Leach, M. J. Padgett, S. M. Barnett, S. Franke-Arnold, and J. Courtial, Phys. Rev. Lett. 88, 257901 (2002).

11. M. V. Vasnetsov, J. P. Torres, D. V. Petrov, and L. Torner, Opt. Lett. 28, 2285 (2003).

12. R. Zambrini and S. M. Barnett, Phys. Rev. Lett. 96, 113901 (2006).

13. C.-S. Guo, S.-J. Yue, and G.-X. Wei, Appl. Phys. Lett. 94, 231104 (2009).

14. G. C. G. Berkhout and M. W. Beijersbergen, Phys. Rev. Lett. 101, 100801 (2008).

15. I. K. Ludlow and J. Everitt, J. Opt. Soc. Am. A 17, 2229 (2000).

16. J. S. Jaffe, Opt. Express 15, 12217 (2007).

17. G. Molina-Terriza, Phys. Rev. A 78, 053819 (2008). 